\title{
Conference Paper \\ Biocompatibility Issues of Next Generation Decellularized Bioprosthetic Devices
}

\author{
Michele Spina, ${ }^{1}$ Filippo Naso, ${ }^{2}$ Irene Zancan, ${ }^{2}$ Laura Iop, ${ }^{2}$ \\ Monica Dettin, ${ }^{3}$ and Gino Gerosa ${ }^{2}$ \\ ${ }^{1}$ Department of Experimental Biomedical Sciences, University of Padova, Viale G. Colombo 3, 35131 Padua, Italy \\ ${ }^{2}$ Department of Cardiac, Thoracic and Vascular Sciences, University Hospital of Padua, Via Giustiniani 2, 35128 Padua, Italy \\ ${ }^{3}$ Department of Industrial Engineering, University of Padova, Via Marzolo 9, 35131 Padua, Italy
}

Correspondence should be addressed to Filippo Naso; fnaso@bio.unipd.it

Received 22 November 2013; Accepted 11 March 2014; Published 12 May 2014

Academic Editors: V. La Carrubba, A. Lepedda, and J. C. Rodriguez-Cabello

This Conference Paper is based on a presentation given by Michele Spina at "LIAC Meeting on Vascular Research 2013" held from 18 September 2013 to 21 September 2013 in Alghero, Italy.

Copyright (c) 2014 Michele Spina et al. This is an open access article distributed under the Creative Commons Attribution License, which permits unrestricted use, distribution, and reproduction in any medium, provided the original work is properly cited.

With respect to the limited lifespan of glutaraldehyde-treated bioprostheses (BHVs) to date there is almost no alternative when heart valve replacement surgery is required and most advanced current research attempts to develop tissue engineered valve scaffolds to be implanted in vivo or after in vitro preconditioning and dynamic seeding with host cells. However the clinical outcomes of grafting detergent-based cell-depleted tissue engineered xenogeneic constructs are still controversial. Insufficient quantitative evaluations performed at preclinical level about the residual content of xenogeneic epitopes, detergents, and nucleic acid materials in such scaffolds have led to disappointing and disastrous results. The risk of these dramatic accidents reoccurring remains very high unless safety and reliable control tools aimed to reach their complete removal, in order to consider tissues biocompatible and suitable for clinical practice.

\section{Introduction}

The increasing occurrence of heart valve insufficiency, following degenerative or rheumatic aetiology, represents a clinical burden with high social and economic relevance in worldwide population and requires as major resolution the surgical approach of valve substitution. Each year more than 290,000 patients go through valve replacement worldwide, and this number is projected to reach 850,000 by the year 2050 , as the average age of the population increases $[1,2]$. Actually commercialized biological heart valve prostheses (BHVs) ameliorated the natural history of the valve pathology, but their performance and endurance are limited in time. The interplay of multiple factors such as chemical interactions, surgical and mechanical factors, material fatigue [3], immunological and foreign body occurrences [4], and finally blood surface exposure leads to the replacement of BHVs after 8 to 10 years following the original implant.
The limited lifespan of BHVs and availability of human tissue substitutes has led biomedical researchers to explore the use of animal donors as an attractive and unlimited source for biological devices (xenotransplantation) in the development of viable and functional tissue engineered construct for lifelasting prostheses.

To ensure biocompatibility when implanting animal tissues into humans, it is mandatory to avoid hyperacute and acute rejection and vascular injury by removing the resident cellular component, antigens, and nucleic acids remnants. To meet these criteria, tissues are subjected to decellularization procedures combining the action of physical agents, detergents, enzymes, and chemical compounds under controlled conditions and without adversely affecting the architecture, ultrastructure, mechanical integrity, and biological activity of the residual extracellular matrix (ECM) [5].

Nevertheless, when implanted in vivo, a decellularized xenogeneic ECM has been shown to maintain the potential 
to attract inflammatory cells and to induce platelet activation with controversial clinical outcomes [6-9]. Xenograft biomimetism is directly related to the presence of several antigens including the notorious alpha-gal epitope [10]. Such antigen resulted to be expressed in most mammalian tissues, except humans and higher primates. In humans, the continuous antigenic stimulation by gastrointestinal flora (expressing the alpha-gal epitope) results in the production of antialpha-gal antibodies accounting for 1 to $3 \%$ of the circulating immunoglobulins [11]. The residual presence of alpha-gal xenoantigen in current commercial BHVs [12] significantly increases the human anti-galactose titers, starting from day 10 following implantation [13] and reaching a peak at around 3 months [14].

Actually the efficacy of a decellularization treatment could be evaluated by several methods including classical histological techniques, immunohistochemistry and/or -fluorescence analysis, and DNA quantification. Strangely enough, the detection and quantification of residual alphagal and/or alpha-gal competitor antigens have not yet been adopted as a standardised control procedure for the production of reliable xenogeneic materials suitable for human implantation.

Since the alpha-gal epitope could remain exposed and reactive in the cellular membrane residues still entrapped between the fibres of the ECM (if not properly removed by the action of detergents), our group has developed a method for evaluating the amount of such antigen before and after different detergent-based decellularization procedures.

\section{Alpha-Gal in Native and Decellularized Porcine Heart Valves}

Ten years ago, detection of alpha-gal epitopes in xenogeneic tissues relied only on the use of alpha-gal reactive lectin molecules, chiefly the Bandeiraea griffonia simplicifolia (GSIB4). Although lectins are not antibodies, for a long time they have been used conjugated with fluorophores for labelling tissue sections. Noteworthy is that lectins feature a reactive group able to specifically interact with the carbohydrate components such as the alpha-gal digalactoside, without excluding affinity for similar groups even if devoid of immunogenic reactivity [17]. To avoid these confounding results, our group strongly based the investigation on the use of the M86 anti-alpha-gal monoclonal antibody [16]. Described for the first time by Galili et al. [18], M86 is an IgM isotype specifically directed toward these xenogeneic sugar residues. In terms of specificity of alpha-gal reactive site recognition the comparison between lectin-based and M86based procedures, performed by our group [15], revealed that in porcine fresh tissue the overlap of fluorescence signals of M86 was limited to $61.5 \pm 2.6 \%$ referred to the total signal obtained adopting lectins. This means that, for native tissues, the use of lectins as alpha-gal detector can lead to an overestimation of the xenogeneic epitopes amount (Figure 1). Conversely, alarming results are obtained by analysis of the same tissue after decellularization treatment. According to the cellular removal procedure developed by Kasimir and
TABLE 1: Amount of alpha-gal expressed as number of epitopes for each $10 \mathrm{mg}$ of wet tissue weight in porcine aortic and pulmonary cusps before (native) and after different detergent-based decellularization treatment ( $n=9$ for each samples treatment). The numbers of epitopes were determined by comparison with a standardized source of alpha-gal (rabbit red blood cells).

\begin{tabular}{lcc}
\hline $\begin{array}{l}\text { Samples } \\
\text { treatment }\end{array}$ & $\begin{array}{c}\text { Amount of alpha-gal } \\
\text { epitope } \\
\text { in porcine aortic } \\
\text { cusp }\end{array}$ & $\begin{array}{c}\text { Amount of alpha-gal } \\
\text { epitope } \\
\text { in porcine pulmonary } \\
\text { cusp }\end{array}$ \\
\hline $\begin{array}{l}\text { Native } \\
\text { Kasimir's }\end{array}$ & $12 * 10^{10} \pm 0.5$ & $19 * 10^{10} \pm 0.4$ \\
$\begin{array}{l}\text { Protocol [19] } \\
\text { Tricol }\end{array}$ & $7.1 * 10^{10} \pm 0.5$ & $6.5 * 10^{10} \pm 0.5$ \\
\hline
\end{tabular}

colleagues [19], our group treated pulmonary and aortic porcine valves with a solution of $0.05 \%$ Triton X-100, $0.05 \%$ sodium deoxycholate, and 0.05\% Igepal CA-630, followed by nuclease digestion. The recognition of the alpha-gal epitopes in such decellularized tissues carried out with the use of the GS-IB4 lectin by Kasimir et al. demonstrated a complete elimination of the antigen in both the wall and the leaflets of the pulmonary valvulated conduit (although no image was provided by the authors to support such findings). Such evaluation was radically different adopting as detector the monoclonal antibody M86. In fact a quantitative ELISA test developed for the dosage of such xenogeneic epitope in native, decellularized, or glutaraldehyde fixed tissues [12, 16] highlighted that $60.01 \%$ and $34.21 \%$ of the original alphagal antigens present in the native tissues (for porcine aortic and pulmonary valve, resp.) were still present and reactive despite the detergent-based decellularization treatment (Table 1). The procedure of Kasimir and colleagues [19] was also compared with a decellularization method developed by our team (named Tricol), based on the combined action of detergents such as Triton X-100, sodium cholate, and nuclease (Benzonase) [20-24]. The alpha-gal immunofluorescence distribution was evaluated with both M86 and GS-IB4 lectin. While no M86 antibody binding was found in Tricol-treated cusps (indicating that the tissue was free from alpha-gal moiety), the whole fibres of the ECM (collagen and elastin) exhibited an unprecedented diffuse and intense affinity for the lectin, with a widespread green fluorescence (Figure 2) [16]. Similar images have been reported by Kasimir et al. even if the authors did not specifically comment about that [19]. The aspecific interactions of the lectin molecules with the ECM components are responsible for the production of a high background noise which makes it impossible to discriminate the presence of the alpha-gal epitopes. Accordingly, the reliability of lectins as alpha-gal detector in decellularized tissues appears to be questionable suggesting avoiding such procedure.

\section{Other Unwanted Leftovers of the Decellularization Process}

Cellular remnants, belonging to dead cells (i.e., membrane debris), are able to activate innate and acquired immune 


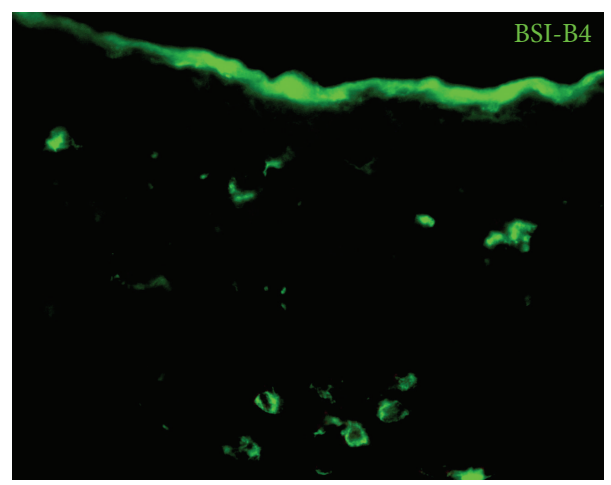

(a)

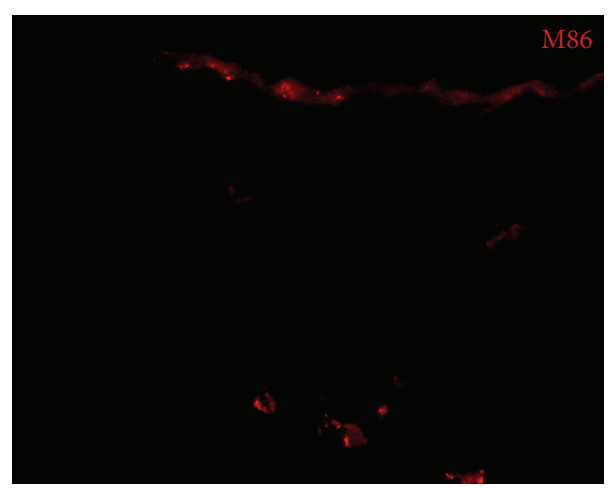

(b)

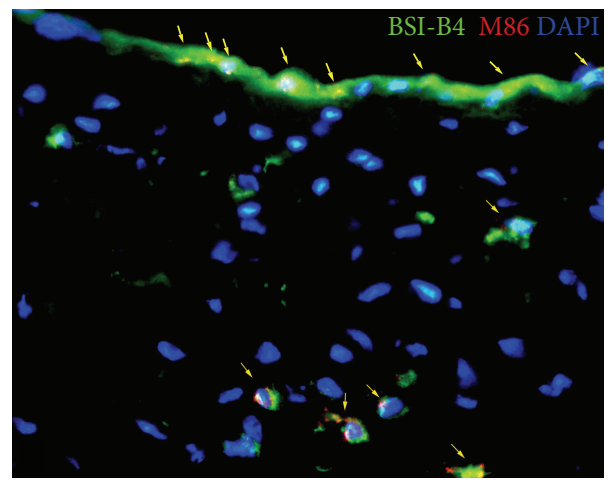

(c)

FIGURE 1: Alpha-gal contextual assessment in native porcine pulmonary cusp using GS-IB4 ((a), green) and M86 monoclonal antibody ((b), red). M86 and GS-IB4 colocalisation signals were highlighted by yellow arrows in (c) pictures. In (c) pictures cell nuclei were identified in blue. Magnification $100 \mathrm{x}$. Image previously published in Naso et al. [15].

responses, resulting in an acute failure of the implanted graft or in a chronic inflammatory reaction [25]. In particular, antigenic determinants exposed on the cells surface may persist in membrane residues and be still capable of reacting notwithstanding the elimination of each single whole-structured cell. Therefore the removal of the alphagal epitope, physiologically present on the surface of the cell membrane of xenogeneic tissues, is likely to be assumed as an index for the assessment of complete cellular debris removal.

While the alpha-gal-free feature can be considered as a sign of efficient decellularization treatment (confirming the absence of cell membrane remnants), also the presence of nucleic acids residues should be taken into consideration.
In fact, they are liable for the initiation of the calcification process, due to the presence of charged phosphoric acid ester groups similarly as in the case of cell membranes phosphate groups $[6,7]$. Finally, a key role in the production of a nonantigenic acellularized matrix is played by the detergent. Such chemical compounds are able to dissolve cell membranes but they have also a high cytotoxic potential further to the ability to trigger the immune system (they are used as adjuvant for the manufacturing of vaccines [26]). Current investigation present in the literature, limited to the evaluation of the detergent release from the treated tissue $[27,28]$, demonstrates how, despite repeated washing, it is not possible to completely eliminate all traces of surfactant 


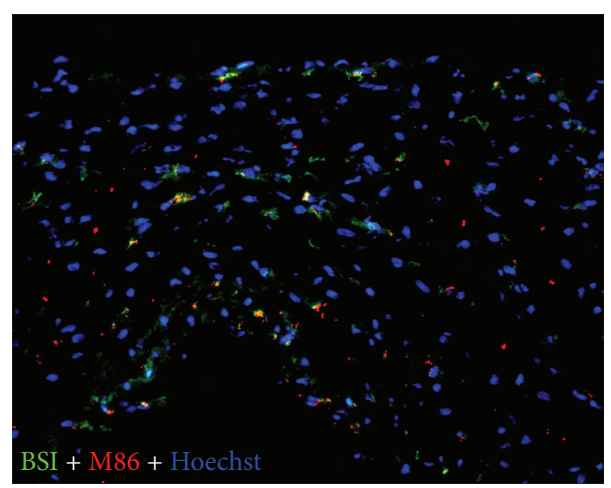

(a)

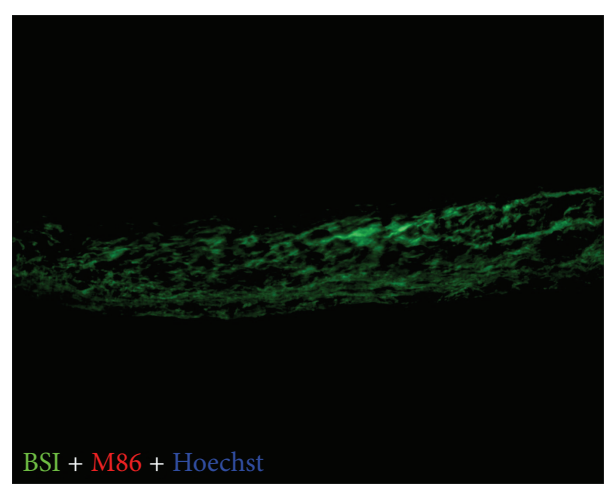

(b)

FIGURE 2: Alpha-gal comparative detection with GS-IB4 isolectin and M86 antibody. Alpha-gal epitopes revealed by immunofluorescence for M86 antibody (red) and GS-IB4 isolectin costaining (green) in native conditions (a). After TriCol decellularization (b), epitopes are no longer immunologically detectable by M86, while GS-IB4 reveals an unspecific attraction to matrix fibers. Note in (b) the full absence of Hoechst signal (blue), meaning an effective decellularization with total removal of nuclear material. Scale bar: $100 \mu \mathrm{m}$. Image previously published in Naso et al. [16].

from the treated biologic material. Residues of detergent are able to decrease the tensile strength of elastin fibers, greatly enhancing the susceptibility of insoluble elastin to proteolysis [29-32] with subsequent structural degeneration. Moreover, the physiochemical properties of some detergents such as the derivatives of bile salts (cholate and deoxycholate salt) are strongly dependent on temperature and $\mathrm{pH}$ conditions. Changes of these parameters are responsible for the transition to a gel state of the detergent solution, remaining trapped within the ECM and making it difficult to wash from the tissue. Such occurrence is aggravated when, within this gel, residues of cell membranes may be present and expose the alpha-gal antigen that making the treated tissue highly immunogenic. Actually our team is committed to the first detergent residues determination performed directly on the resulting treated tissue scaffold. Preliminary data seem to demonstrate how sodium deoxycholate is the surfactant most difficult to remove, accumulating within the insoluble tissue matrix in nonnegligible amount.

\section{Conclusion}

Insufficient quantitative evaluations performed at preclinical level about the residual content of xenogeneic epitopes, detergents, and nucleic acid materials have led to disappointing and disastrous results [7-9]. The risk of these dramatic accidents reoccurring remains very high unless safety and reliable control tools are adopted, particularly aimed at the complete removal of major xenogeneic determinants (alphagal) and calcification-prone nucleic acid residues. All of these criteria are likely to be strictly fulfilled in order to consider the tissue biocompatible and suitable for clinical practice.

\section{Conflict of Interests}

The authors declare that there is no conflict of interests regarding the publication of this paper.

\section{Acknowledgments}

This research was supported by Fondazione Cassa di Risparmio di Padova e Rovigo (Italian Heart Project). The authors would like to thank all the LIAC (Latinorum Investigatorum de Arteriis Colloquium) scientific committee.

\section{References}

[1] K. Kuwaki, Y.-L. Tseng, F. J. M. F. Dor et al., "Heart transplantation in baboons using $\alpha 1,3$-galactosyltransferase gene-knockout 
pigs as donors: initial experience," Nature Medicine, vol. 11, no. 1, pp. 29-31, 2005.

[2] B. Bertipaglia, F. Ortolani, L. Petrelli et al., "Cell characterization of porcine aortic valve and decellularized leaflets repopulated with aortic valve interstitial cells: the VESALIO project (vitalitate exornatum succedaneum aorticum labore ingenioso obtenibitur)," The Annals of Thoracic Surgery, vol. 75, no. 4, pp. 1274-1282, 2003.

[3] S. Gabbay, P. Kadam, S. Factor, and T. K. Cheung, "Do heart valve bioprostheses degenerate for metabolic or mechanical reasons?" The Journal of Thoracic and Cardiovascular Surgery, vol. 95, no. 2, pp. 208-215, 1988.

[4] C. S. Park, S.-S. Park, S. Y. Choi, S. H. Yoon, W.-H. Kim, and Y. J. Kim, "Anti alpha-gal immune response following porcine bioprosthesis implantation in children," The Journal of Heart Valve Disease, vol. 19, no. 1, pp. 124-130, 2010.

[5] T. W. Gilbert, T. L. Sellaro, and S. F. Badylak, "Decellularization of tissues and organs," Biomaterials, vol. 27, no. 19, pp. 3675$3683,2006$.

[6] M.-T. Kasimir, E. Rieder, G. Seebacher et al., "Decellularization does not eliminate thrombogenicity and inflammatory stimulation in tissue-engineered porcine heart valves," The Journal of Heart Valve Disease, vol. 15, no. 2, pp. 278-286, 2006.

[7] P. Simon, M. T. Kasimir, G. Seebacher et al., "Early failure of the tissue engineered porcine heart valve SYNERGRAFT in pediatric patients," European Journal of Cardio-Thoracic Surgery, vol. 23, no. 6, pp. 1002-1006, 2003.

[8] A. Rüffer, A. Purbojo, I. Cicha et al., "Early failure of xenogenous de-cellularised pulmonary valve conduits-a word of caution!"” European Journal of Cardio-Thoracic Surgery, vol. 38, no. 1, pp. 78-85, 2010.

[9] I. Voges, J. H. Bräsen, A. Entenmann et al., "Adverse results of a decellularized tissue-engineered pulmonary valve in humans assessed with magnetic resonance imaging," European Journal of Cardio-Thoracic Surgery, vol. 44, no. 4, pp. e272-e279, 2013.

[10] B. A. Macher and U. Galili, "The Gal $\alpha 1,3 \mathrm{Gal} \beta 1,4$ GlcNAc-R ( $\alpha$ Gal) epitope: a carbohydrate of unique evolution and clinical relevance," Biochimica et Biophysica Acta: General Subjects, vol. 1780, no. 2, pp. 75-88, 2008.

[11] R. Rieben, N. V. Bovin, E. Y. Korchagina et al., "Xenotransplantation: in vitro analysis of synthetic $\alpha$-galactosyl inhibitors of human anti-Gal $\alpha 1 \rightarrow 3 \mathrm{Gal}$ IgM and IgG antibodies," Glycobiology, vol. 10, no. 2, pp. 141-148, 2000.

[12] F. Naso, A. Gandaglia, T. Bottio et al., "First quantification of alpha-Gal epitope in current glutaraldehyde-fixed heart valve bioprostheses.," Xenotransplantation, vol. 20, no. 4, pp. 252-261, 2013.

[13] K. Z. Konakci, B. Bohle, R. Blumer et al., "Alpha-Gal on bioprostheses: xenograft immune response in cardiac surgery," European Journal of Clinical Investigation, vol. 35, no. 1, pp. 1723, 2005.

[14] A. Mangold, T. Szerafin, K. Hoetzenecker et al., "Alpha-Gal specific IgG immune response after implantation of bioprostheses," The Thoracic and Cardiovascular Surgeon, vol. 57, no. 4, pp. 191195, 2009.

[15] F. Naso, A. Gandaglia, L. Iop, M. Spina, and G. Gerosa, "Alpha-Gal detectors in xenotransplantation research: a word of caution," Xenotransplantation, vol. 19, no. 4, pp. 215-220, 2012.

[16] F. Naso, A. Gandaglia, L. Iop, M. Spina, and G. Gerosa, "First quantitative assay of alpha-Gal in soft tissues: presence and distribution of the epitope before and after cell removal from xenogeneic heart valves," Acta Biomaterialia, vol. 7, no. 4, pp. 1728-1734, 2011.

[17] M. Agostino, M. S. Sandrin, P. E. Thompson, E. Yuriev, and P. A. Ramsland, "Identification of preferred carbohydrate binding modes in xenoreactive antibodies by combining conformational filters and binding site maps," Glycobiology, vol. 20, no. 6, pp. 724-735, 2010.

[18] U. Galili, D. C. Latemple, and M. Z. Radic, "A sensitive assay for measuring $\alpha$-Gal epitope expression on cells by a monoclonal anti-Gal antibody," Transplantation, vol. 65, no. 8, pp. 1129-1132, 1998.

[19] M.-T. Kasimir, E. Rieder, G. Seebacher, E. Wolner, G. Weigel, and P. Simon, "Presence and elimination of the xenoantigen Gal $(\alpha 1,3) \mathrm{Gal}$ in tissue-engineered heart valves," Tissue Engineering, vol. 11, no. 7-8, pp. 1274-1280, 2005.

[20] M. Spina, F. Ortolani, A. El Messlemani et al., "Isolation of intact aortic valve scaffolds for heart-valve bioprostheses: extracellular matrix structure, prevention from calcification, and cell repopulation features," Journal of Biomedical Materials Research A, vol. 67, no. 4, pp. 1338-1350, 2003.

[21] L. Iop, V. Renier, F. Naso et al., "The influence of heart valve leaflet matrix characteristics on the interaction between human mesenchymal stem cells and decellularized scaffolds," Biomaterials, vol. 30, no. 25, pp. 4104-4116, 2009.

[22] T. Bottio, V. Tarzia, C. D. Lin et al., "The changing hydrodynamic performance of the decellularized intact porcine aortic root: considerations on in-vitro testing," The Journal of Heart Valve Disease, vol. 19, no. 4, pp. 485-491, 2010.

[23] F. Naso, A. Gandaglia, M. Formato et al., "Differential distribution of structural components and hydration in aortic and pulmonary heart valve conduits: impact of detergent-based cell removal," Acta Biomaterialia, vol. 6, no. 12, pp. 4675-4688, 2010.

[24] M. Gallo, F. Naso, H. Poser et al., "Physiological performance of a detergent decellularized heart valve implanted for 15 months in Vietnamese pigs: surgical procedure, follow-up, and explant inspection," Artificial Organs, vol. 36, no. 6, pp. E138-E150, 2012.

[25] S. Arai and E. C. Orton, "Immunoblot detection of soluble protein antigens from Sodium dodecyl Sulfate- and Sodium deoxycholate-treated candidate bioscaffold tissues," The Journal of Heart Valve Disease, vol. 18, no. 4, pp. 439-443, 2009.

[26] J. H. Playfair and J. B. de Souza, "Vaccination of mice against malaria with soluble antigens. I. The effect of detergent, route of injection, and adjuvant," Parasite Immunology, vol. 8, no. 5, pp. 409-414, 1986.

[27] S. Caamaño, A. Shiori, S. H. Strauss, and E. C. Orton, "Does Sodium dodecyl Sulfate wash out of detergent-treated bovine pericardium at cytotoxic concentrations?" The Journal of Heart Valve Disease, vol. 18, no. 1, pp. 101-105, 2009.

[28] S. Cebotari, I. Tudorache, T. Jaekel et al., "Detergent decellularization of heart valves for tissue engineering: toxicological effects of residual detergents on human endothelial cells," Artificial Organs, vol. 34, no. 3, pp. 206-210, 2010.

[29] H. M. Kagan, G. D. Crombie, R. E. Jordan, W. Lewis, and C. Franzblau, "Proteolysis of elastin-ligand complexes. Stimulation of elastase digestion of insoluble elastin by Sodium dodecyl Sulfate," Biochemistry, vol. 11, no. 18, pp. 3412-3418, 1972.

[30] R. E. Jordan, N. Hewitt, W. Lewis, H. Kagan, and C. Franzblau, "Regulation of elastase catalyzed hydrolysis of insoluble elastin by synthetic and naturally occurring hydrophobic ligands," Biochemistry, vol. 13, no. 17, pp. 3497-3503, 1974. 
[31] V. Guantieri, A. M. Tamburro, and D. Daga Gordini, "Conformational changes induced in $\alpha$-elastin by cholesterol, taurocholate and unsaturated fatty acids," International Journal of Biological Macromolecules, vol. 2, no. 2, pp. 68-72, 1980.

[32] V. Guantieri, A. M. Tamburro, and D. Daga Gordini, "Interactions of human and bovine elastins with lipids: their proteolysis by elastase," Connective Tissue Research, vol. 12, no. 1, pp. 79-83, 1983. 


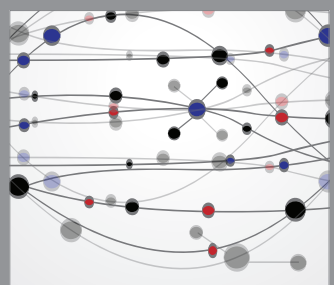

The Scientific World Journal
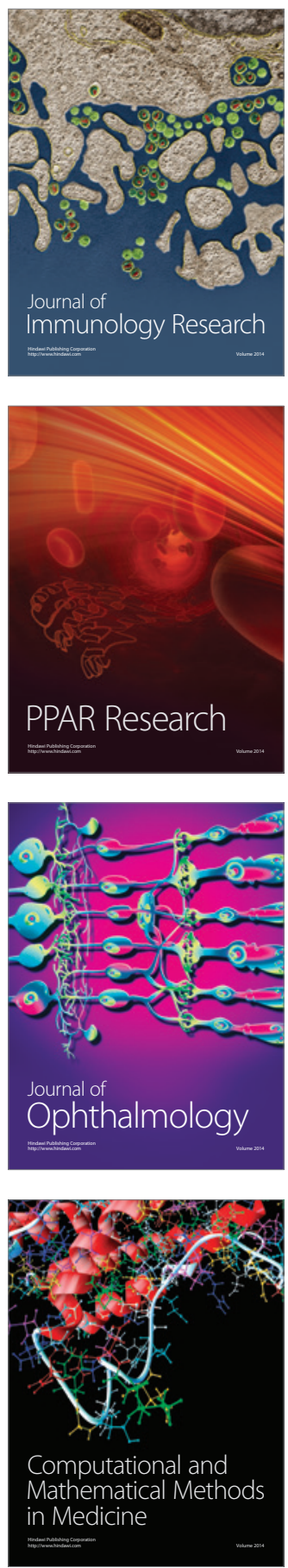

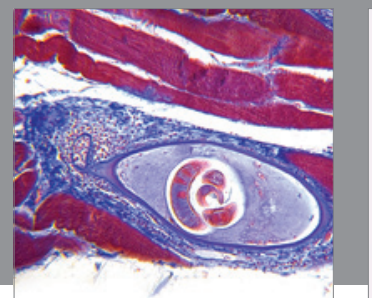

Gastroenterology

Research and Practice
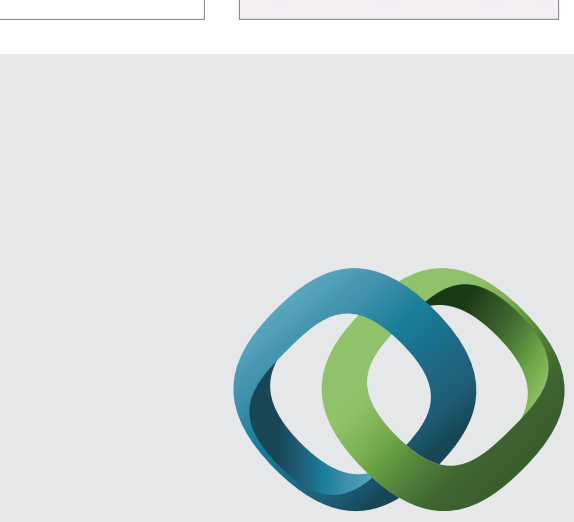

\section{Hindawi}

Submit your manuscripts at

http://www.hindawi.com
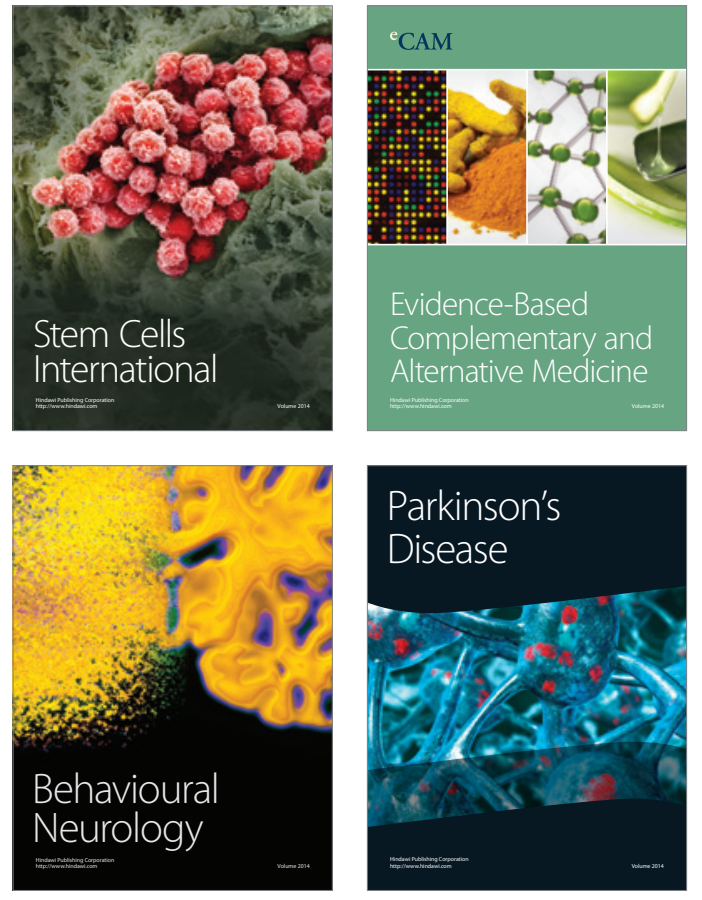
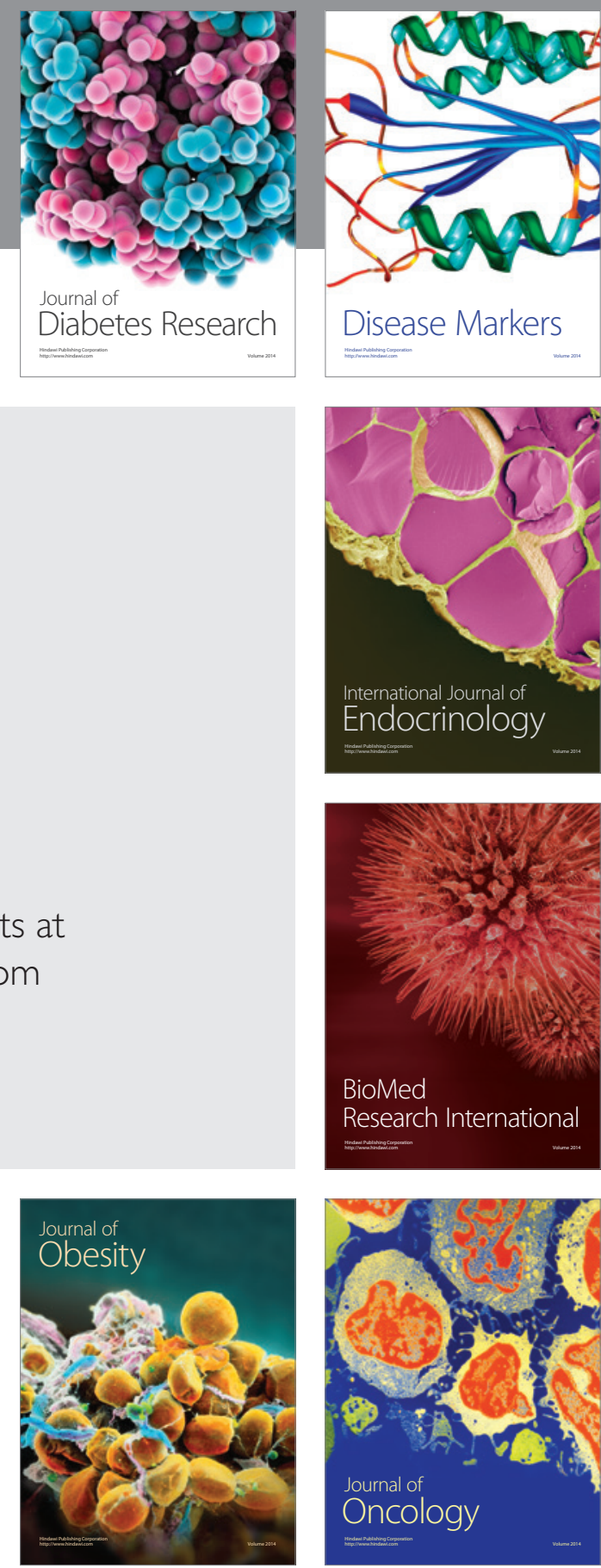

Disease Markers
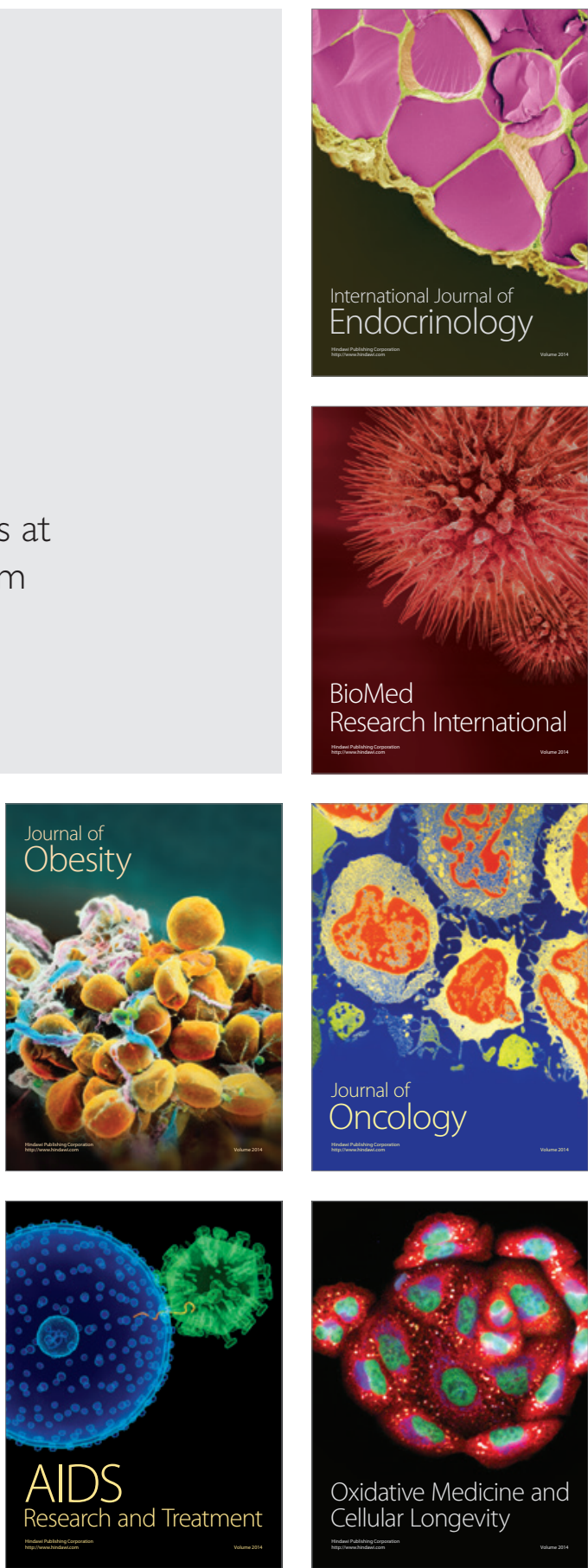